\title{
Performance and Application of Chromatic/Spherical Aberration-Corrected 30 kV Transmission Electron Microscope
}

\author{
T. Sasaki ${ }^{*}$, H. Sawada ${ }^{*}$, F. Hosokawa ${ }^{*}$, Y. Shimizu ${ }^{*}$, T. Nakamichi ${ }^{*}$, S. Yuasa ${ }_{* * *}$, \\ M. Kawazoe ${ }^{*}$, T. Kaneyama ${ }^{*}$ Y. Kondo ${ }^{*}$, K. Kimoto ${ }^{* *}$ and K. Suenaga ${ }^{* * *}$ \\ *JEOL Ltd., 3-1-2 Musashino, Akishima Tokyo 196-8558, Japan \\ ${ }_{* * *}^{*}$ National Institute for Materials Science (NIMS), 1-1, Namiki, Tsukuba, Ibaraki, 305-0044, Japan \\ *** Nanotube Research Center, National Institute of Advanced Industrial Science and Technology \\ (AIST), Central 5, 1-1-1 Higashi, Tsukuba, Ibaraki 305-8565, Japan
}

For characterization of carbon materials such as graphenes and carbon nanotubes, it is effective to apply a lower acceleration voltage to observe them free from knock-on damage originated from a probing electron beam on the specimen. Since a wavelength increases at a lower acceleration voltage, compensation of geometrical aberrations is important to yield atomic resolution. Compensating both spherical aberration (Cs) and six-fold astigmatism (A6) with a delta-type Cs corrector [1], we have achieved atomic resolution imaging [2] and succeeded in atom-by-atom spectroscopy of carbon materials $[3,4]$ at $30-60 \mathrm{kV}$ in a Triple $\mathrm{C} \# 1$ microscope. For a next challenging target to further improve resolution at a lower acceleration voltage, we have uniquely developed a new type of chromatic aberration (Cc) corrector [5], and installed it on a $30 \mathrm{kV}$ transmission electron microscope (TEM) with a delta-type Cs corrector. This paper reports results of performance tests and application data of Cc/Cs-corrected $30 \mathrm{kV}$-TEM.

Figure 1 shows a photograph of a developed microscope (Triple C \#2). A Schottky field emission gun (FEG) is installed on the top of the microscope column and is to be replaced to a cold FEG to improve coherency of an electron source. A platform of the microscope is a JEM-ARM200F. Cc and Cs correctors, which were arranged in tandem, were installed between an objective lens and an intermediate lens. Using the effect of combination concave lens, generated from a thick electrostatic or magnetic quadrupole (two-fold astigmatism) field, Cc was compensated. Then residual (geometrical) aberrations were compensated with a delta corrector. All results reported here were taken at $30 \mathrm{kV}$. Figure 2 (a) and (b) show a Cc/Cs-corrected high-resolution TEM (HRTEM) image of $\operatorname{Si}[110]$ and its power spectrum. Lattice fringes of $\{002\}$ and $\{220\}$, corresponding to spacings of $272 \mathrm{pm}$ and $192 \mathrm{pm}$, respectively, were clearly imaged in the TEM image. Clear spots of $125 \mathrm{pm}$ and $105 \mathrm{pm}$ can be seen in the power spectrum. Figure 3 (a) and (b) show a Cc/Cs-corrected HRTEM image of $\mathrm{SrTiO}_{3}[001]$ and its power spectrum. Lattice fringes of $\{110\}$ and $\{200\}$, corresponding to spacings of $276 \mathrm{pm}$ and $195 \mathrm{pm}$, respectively, were clearly imaged in the TEM image. Clear spots of $124 \mathrm{pm}$ and $98 \mathrm{pm}$ was observed in the power spectrum. These results demonstrate the stability of the $\mathrm{Cc} / \mathrm{Cs}$-corrected microscope at $30 \mathrm{kV}$ and other application data will be reported.

\section{References}

[1] H. Sawada et al., J. Electron Microsc. 58 (2009) 341.

[2] T. Sasaki et al., J. Electron Microsc. 59 (2010) S7.

[3] K. Suenaga et al., Nature Chem. 1 (2009) 415.

[4] K. Suenaga and M. Koshino, Nature 468 (2010) 1088.

[5] H. Sawada et al., IMC 17 proceedings (2010) I20.8; T. Sasaki et al, ibid. (2010) I2.2.

[6] This work was supported by Japan Science and Technology Agency under the CREST project. 


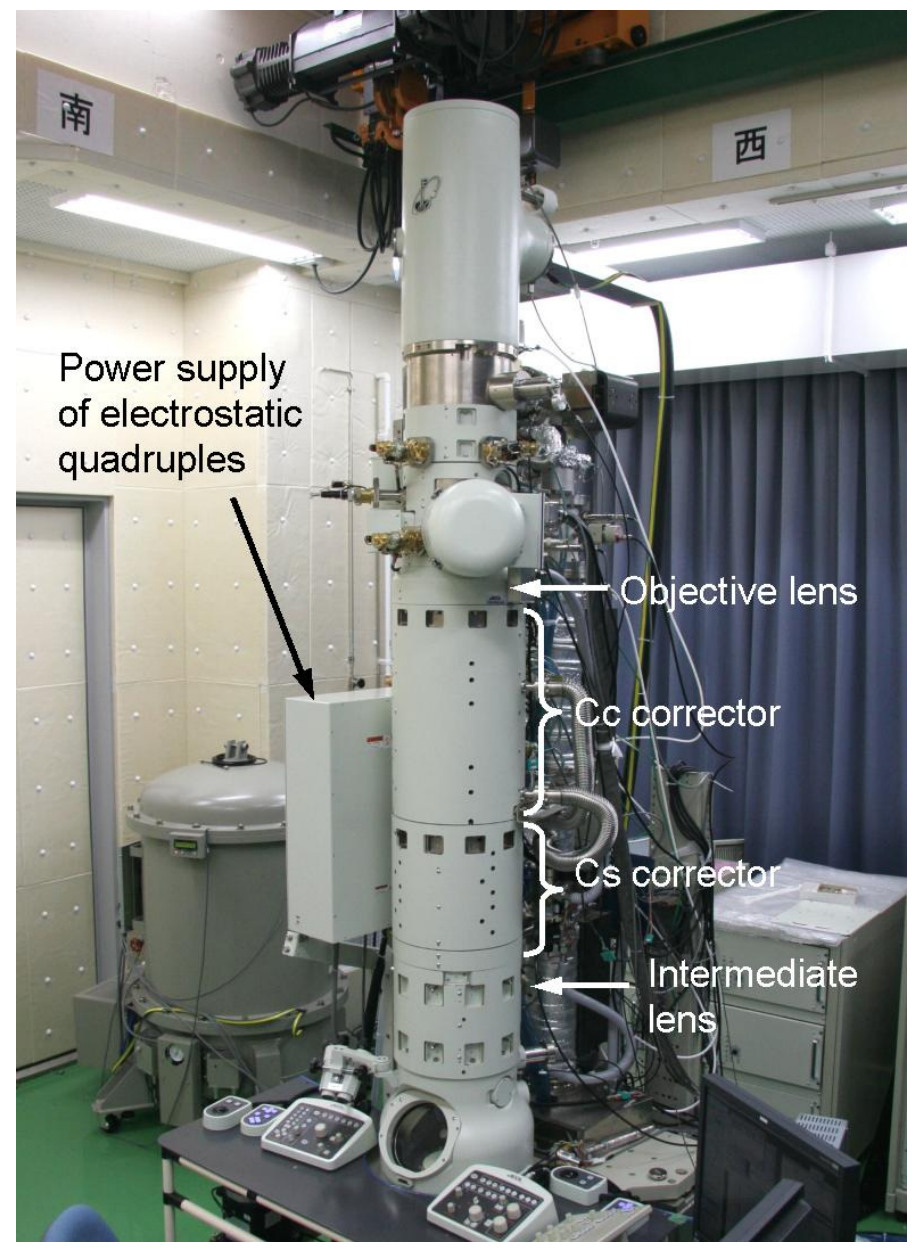

Fig.1 (a) Developed 30 kV TEM (Triple C \#2) with delta-type $\mathrm{Cs}$ and new type of Cc correctors.
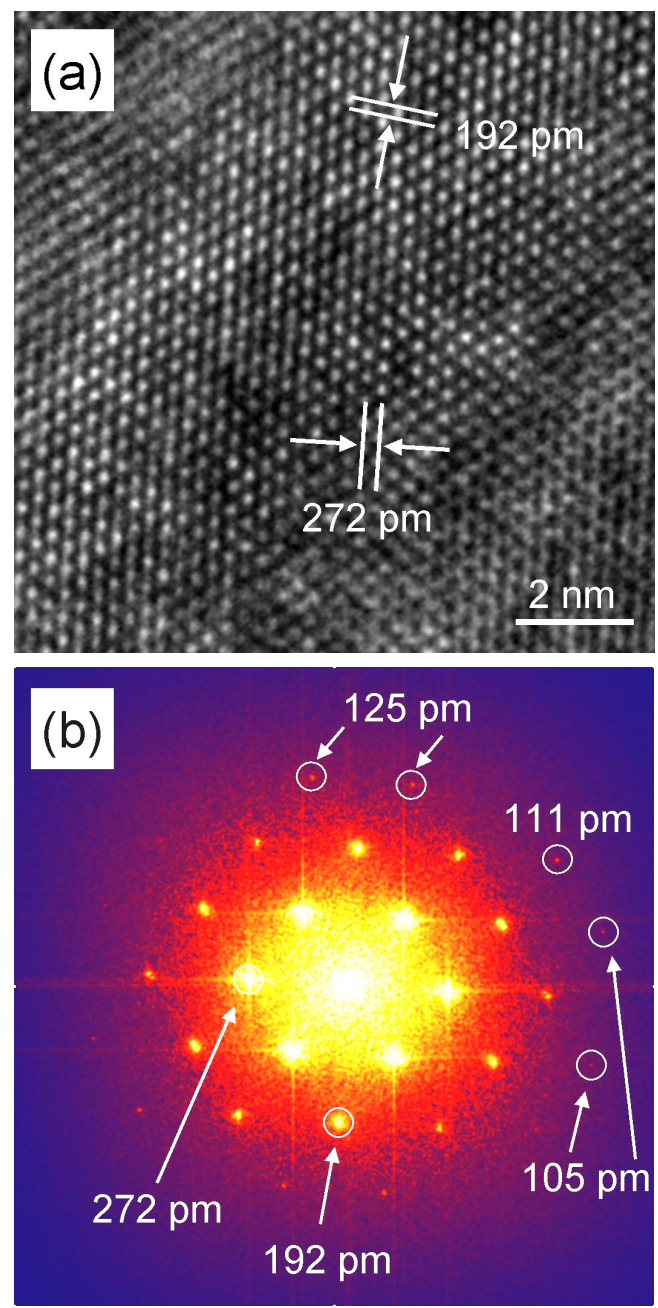

Fig.2 (a) Cc/Cs-corrected HRTEM image (raw data) of $\mathrm{Si}[110]$ taken at $30 \mathrm{kV}$ and (b) its power spectrum.
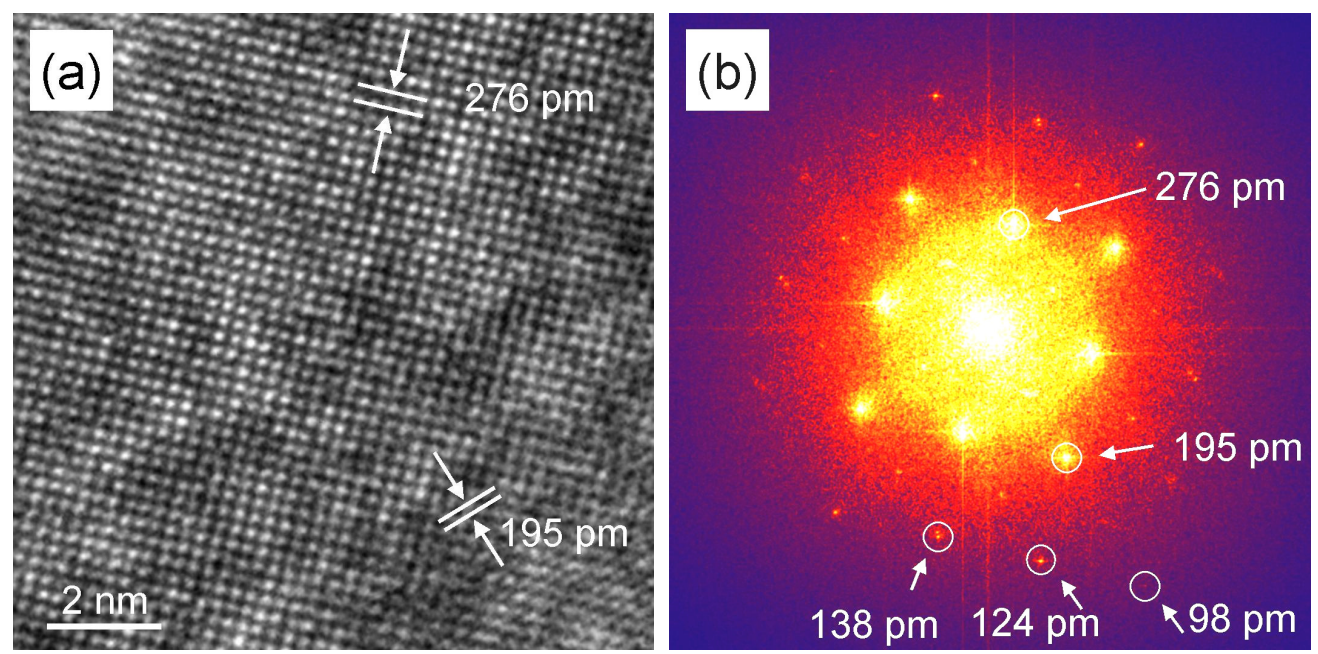

Fig.3 (a) Cc/Cs-corrected HRTEM image (raw data) of $\mathrm{SrTiO}_{3}[001]$ taken at $30 \mathrm{kV}$ and (b) its power spectrum. 